\section{JTI}

JOURNAL OF

TRAUMA AND INJURY

\title{
Reconstruction of a Large Infected Midline Abdominal Wall Defect Using a Latissimus Dorsi Free Flap
}

\author{
Han Gyu Cha, M.D. ${ }^{1}$, Eun Key Kim, M.D., Ph.D. ${ }^{1}$, Suk-Kyung Hong, M.D., Ph.D. ${ }^{2}$ \\ ${ }^{1}$ Department of Plastic Surgery, Asan Medical Center, University of Ulsan College of Medicine, \\ Seoul, Korea \\ ${ }^{2}$ Division of Trauma and Surgical Critical Care, Department of Surgery, Asan Medical Center, \\ University of Ulsan College of Medicine, Seoul, Korea
}

Received: July 5, 2018

Revised: July 23, 2018

Accepted: July 25, 2018

\section{Correspondence to}

Eun Key Kim, M.D., Ph.D.

Department of Plastic Surgery, Asan Medical Center, 88 Olympic-ro 43-gil, Songpa-gu, Seoul 05505, Korea

Tel: $+82-2-3010-3600$

Fax: +82-2-476-7471

E-mail: nicekek@korea.com
Managing large infected midline abdominal defects are clinically challenging and technically demanding. The alloplastic materials, regional flaps, and component separation are usually infeasible because of the size, location, depth, and state of the defects. In these cases, the free flap is the only option with a large well-vascularized tissue that is free to inset regardless of the location. Herein, we report a case of 44-year-old man with a large infected midline abdominal wall defect who was completely treated with a latissimus dorsi myocutaeous free flap followed by negative pressure wound therapy.

Keywords: Abdominal wall; Free flaps; Latissimus dorsi

\section{INTRODUCTION}

Managing complex abdominal wall defects is challenging for both general and plastic surgeons. In cases of large infected defects in particular, the surgeons not only have to understand the anatomy in question but also have to be technically prepared for various reconstruction options. In these situations however, the typically and widely used alloplastic materials should not be employed due to the possibility of reinfection. In addition, surgical techniques such as regional flaps and component separation are typically not feasible in these cases due to the lack of soft tissue. Furthermore, to help control for possible infection of the abdominal wall, a myocutaneous flap is more solid and sturdy than a fasciocutaneous flap [1].

The latissimus dorsi (LD) myocutaneous flap is one of the most widely used as a pedicled or free flap. Its main advantages are the ease of elevation, long vascular ped(http://creativecommons.org/licenses/by-nc/4.0/) which permits unrestricted noncommercial use, distribution, and reproduction in any medium, provided the original work is properly cited. 


\section{JTI}

icle, large diameter of the donor vessels, and the potentially large size of the flap [2]. We here report a case of a successful reconstruction of a large infected midline abdominal wall defect using an LD myocutaneous free flap.

\section{CASE REPORT}

A 44-year-old man was admitted to our hospital with a crushed abdominal wall. An emergency laparotomy revealed a hemoperitoneum with division of the superior mesenteric artery branches, hepatic contusion, spleen contusion, transverse colon tearing, and jejunum perforation. After multiple surgeries to repair the injuries to the small bowel and adjacent organs, the patient was referred to our plastic surgery department with an enterocutaneous fistula 18 months after the trauma. Candida albicans, Candida glabrata, and methicillin resistant Staphylococcus aureus were present in cultures from his wound and

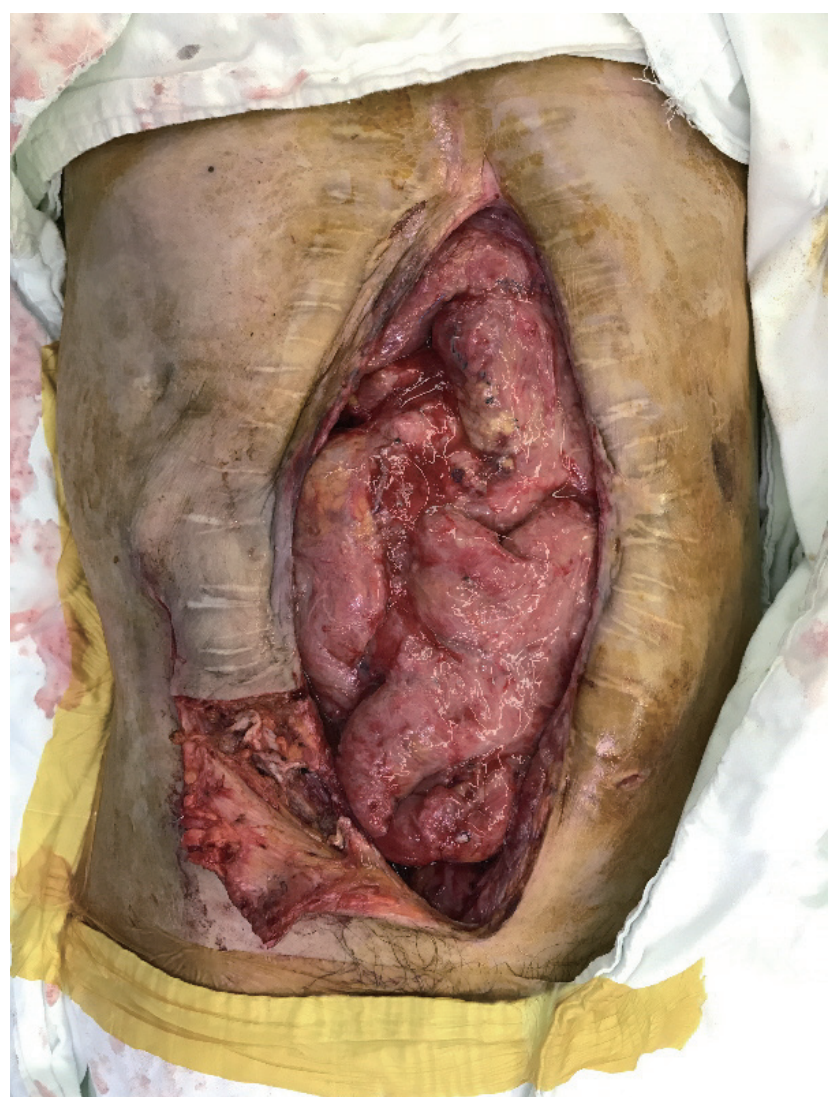

Fig. 1. The $15 \times 30 \mathrm{~cm}^{2}$ sized midline abdominal wall defect in the patient after dissection of the recipient deep inferior epigastric vessels. vancomycin-resistant enterococci were cultured from the patient's stool. The wound was serially debrided and a new duodenojejunostomy was performed in the general surgery department prior to abdominal wall reconstruction. The preoperative abdominal wall defect size was $15 \times 30 \mathrm{~cm}^{2}$ and the use of an LD myocutaneous free flap was planned to cover the defect in combination with a local transposition flap. The deep inferior epigastric artery (DIEA) and vein (DIEV) of the right abdomen were used as the recipient vessels. Fortunately, the DIEA and DIEV were preserved in this patient despite his multiple operations. After elevating a fasciocutaneous flap in the right lower abdomen, the DIEA and DIEV were found beneath the remaining rectus muscle (Fig. 1). The patient was then placed in the lateral position to harvest an LD myocuta-

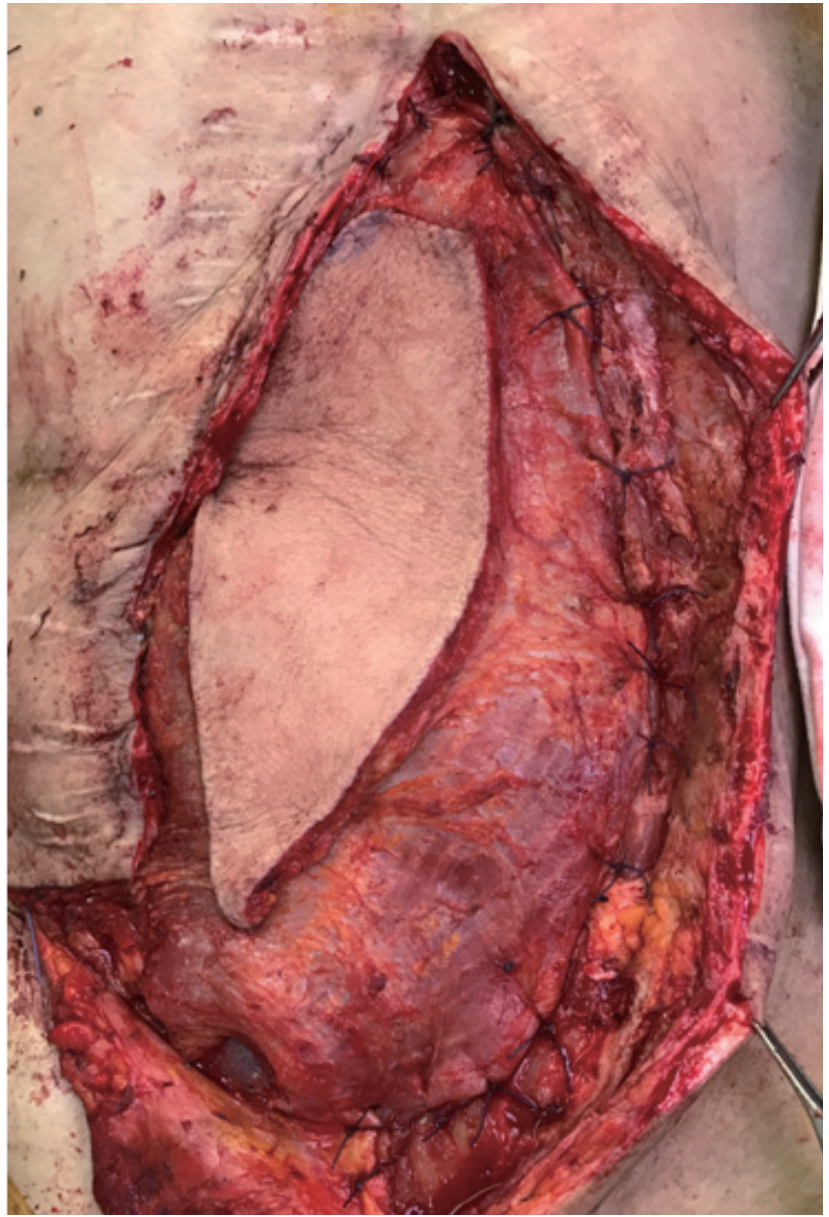

Fig. 2. Intraoperative photograph showing the LD muscle fascia sutured to the remaining deep abdominal fascia to prevent an abdominal hernia and reinforce the abdominal wall. LD: latissimus dorsi. 
neous flap of $10 \times 20 \mathrm{~cm}^{2}$ along with a thoracodorsal artery (TDA) and vein (TDV) of as much length as possible to obtain a sufficient pedicle length for anastomosis. The donor site was primarily closed. Anastomosis was subsequently performed between the DIEA and TDA, and the DIEV and TDV, in the supine position. The pre-elevated fasciocutaneous flap was then transpositioned to the left abdominal wall to cover the lower abdomen defect. The LD muscle fascia was sutured to the remaining deep abdominal fascia to prevent an abdominal hernia and reinforce the abdominal wall (Figs. 2, 3).

Although the LD free flap was healthy and did not cause any complications by itself, a minimal amount of leakage persisted from the duodenal stump and there was discharge from the wound margin after 3 days postoperatively. Negative pressure wound therapy (NPWT) was applied at the site for three weeks after which the wound was fully healed. There was no recurrent leakage or

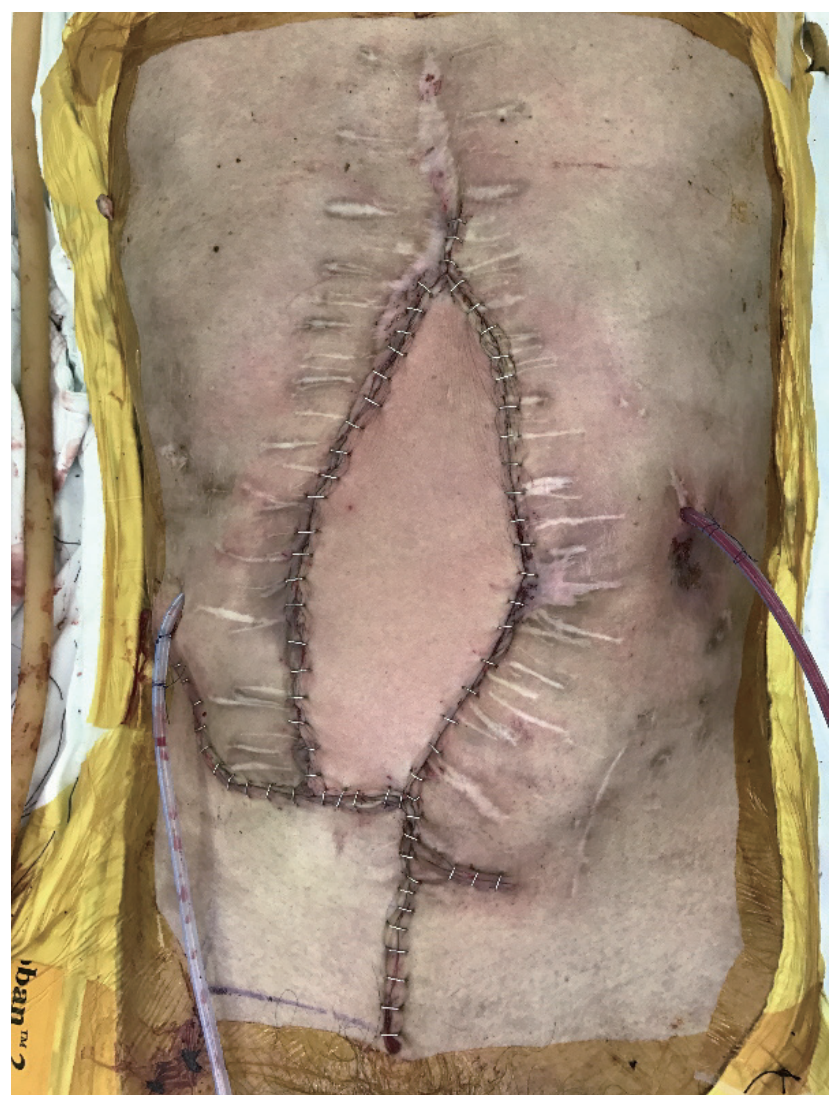

Fig. 3. Immediate postoperative view of the patient's abdomen completely covered with the LD myocutaneous free flap and local transposition flap. LD: latissimus dorsi. wound disruption at the 13-month follow-up with good abdominal support (Figs. 4, 5).

\section{DISCUSSION}

Reconstruction options for abdominal wall defects vary depending on the depth, size, and location of the defect. In complete full-thickness large defects, tissue transfer such as a muscle flap with skin graft or a myocutaneous flap may be the only immediate surgical option [3]. If a full-thickness large defect is located at the midline of the abdomen, however, the treatment options become more limited because local flaps have a restricted arc of rotation and the distance to advance is too long for flaps from the lateral part of the abdomen. The free flap is therefore the best option in these cases. Compared with a local flap, a free flap has the advantage of providing a large volume of well-vascularized tissue with less restriction in terms

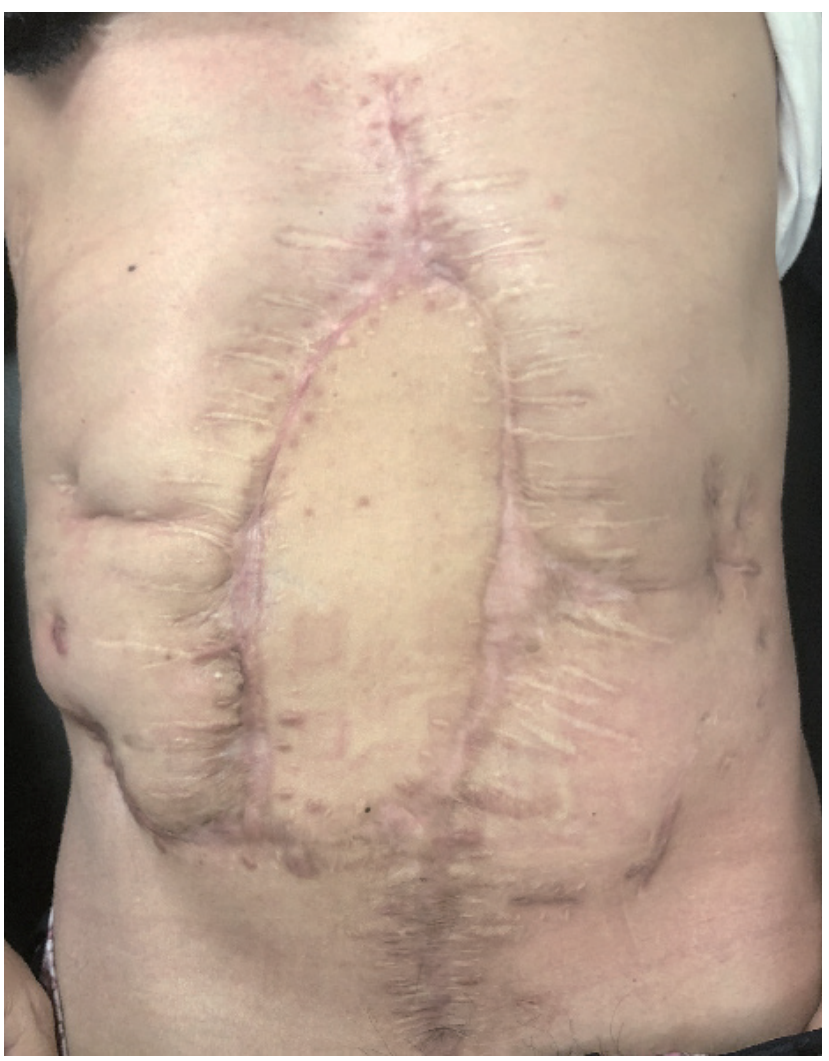

Fig. 4. Postoperative view of the patient's abdomen at 13 months after the reconstruction showing no signs of wound infection or dehiscence. 


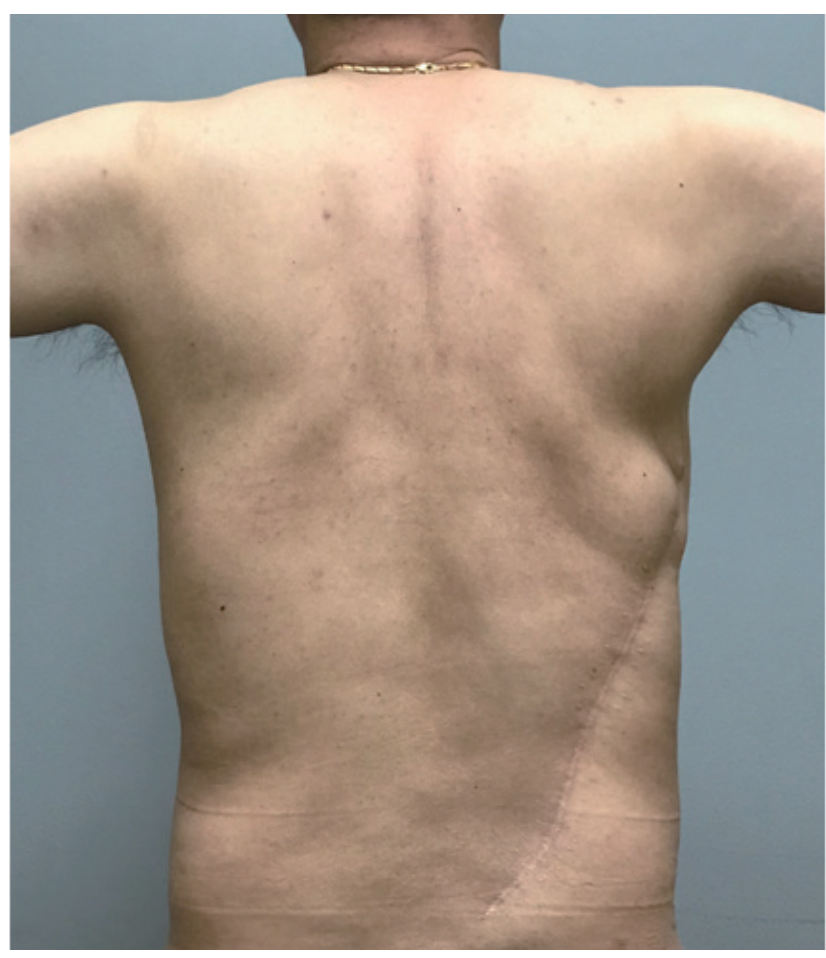

Fig. 5. Postoperative view of the back donor site at 13 months after the reconstruction.

of pedicle reach. The indications for free flap abdominal wall reconstruction reported to date include (a) patients with full thickness moderate to large midline defects that preclude the use of component separation or where the rectus abdominis and its fascia sheath are unavailable; (b) contamination or infection in which the use of totally autologous tissue is preferred but suitable regional muscle flaps are absent; and (c) cases of previously failed reconstructions with alloplastic materials $[4,5]$.

The anterolateral thigh (ALT) flap is one of the most commonly used free flaps for an abdominal wall reconstruction since it can be harvested with the iliotibial tract and tensor fascia lata that can be used to reconstruct a strong alternative fascial layer at the abdominal wall. However, the harvesting of the iliotibial tract and tensor fascia lata can result in a mild deficiency in quadriceps femoris muscle contraction and limited knee joint movement [6]. In addition, the ability to use an ALT flap remains questionable in cases of infected defects. This is because a myocutaneous flap is known to have superior bacterial suppression ability compared with a fasciocu- taneous flap due to rapid early augmentation of muscle blood flow [7]. The myocutaneous flap that can be selected in these cases is the LD flap which can be harvested with a large portion of LD muscle that can cover the defect and also control for possible infection. The donor site is generally primarily closed and well hidden under clothing. The pedicle length is relatively shorter than that in the ALT flap but is sufficient to reach the recipient vessels for anastomosis. In addition, the diameter of the thoracodorsal vessel is large enough for easier anastomosis. By virtue of these advantages, the LD myocutaneous flap has now been used widely in every part of the body with various modifications [8].

The recipient vessels for abdominal free flaps can be classified as intraperitoneal and extraperitoneal. The most popular extraperitoneal recipient vessels used include inferior epigastric vessels, superficial circumflex vessels, internal mammary vessels, and superior epigastric vessels. The gastroepiploic vessels are well known intraperitoneal vessels [9]. We prefer to use extraperitoneal vessels as they are easy to find and dissect without interfering with internal organs. The DIEA used in the current study patient is usually preserved after multiple abdominal surgeries because of its low anatomical location. The only issue with the use of this artery is that a vein graft can be needed if the pedicle length is short.

NPWT has many advantages for use with abdominal defects. It can be employed to remove edema of the abdominal wall and bowel, reduce the size of an open wound, and serve as a temporary closure to control abdominal content [10]. Many surgeons use this technique prior to reconstruction surgery because of these advantages. In our current study case, we utilized NPWT as an adjuvant therapy after the free flap surgery to control for possible bowel leakage. We were able to completely stop any leakage after 3 weeks with this method with no deleterious impacts on the free flap.

In conclusion, the LD myocutaneous free flap is a potential option for the treatment of large midline-infected abdominal defects. The versatility of this flap offers alternative solutions to correcting abdominal defects when other options such as a regional flap, component separation, and alloplastic materials are inadequate. Furthermore, NPWT can serve as a safe adjuvant tool after free 
flap surgery to control for possible residual bowel leakage.

\section{REFERENCES}

1. Calderon W, Chang N, Mathes SJ. Comparison of the effect of bacterial inoculation in musculocutaneous and fasciocutaneous flaps. Plast Reconstr Surg 1986;77:785-94.

2. Watson JS, Craig RD, Orton CI. The free latissimus dorsi myocutaneous flap. Plast Reconstr Surg 1979;64:299-305.

3. Rohrich RJ, Lowe JB, Hackney FL, Bowman JL, Hobar PC. An algorithm for abdominal wall reconstruction. Plast Reconstr Surg 2000;105:202-16; quiz 217.

4. Porshinsky B, Ramasastry S. Abdominal wall reconstruction with free flaps. Clin Plast Surg 2006;33:269-80.

5. Wong $\mathrm{CH}$, Lin $\mathrm{CH}$, Fu B, Fang JF. Reconstruction of complex abdominal wall defects with free flaps: indications and clinical outcome. Plast Reconstr Surg 2009;124:500-9.

6. Kuo YR, Yeh MC, Shih HS, Chen CC, Lin PY, Chiang YC, et al.
Versatility of the anterolateral thigh flap with vascularized fascia lata for reconstruction of complex soft-tissue defects: clinical experience and functional assessment of the donor site. Plast Reconstr Surg 2009;124:171-80.

7. Gosain A, Chang N, Mathes S, Hunt TK, Vasconez L. A study of the relationship between blood flow and bacterial inoculation in musculocutaneous and fasciocutaneous flaps. Plast Reconstr Surg 1990;86:1152-62; discussion 1163.

8. Kim JT, Kim SW, Youn S, Kim YH. What is the ideal free flap for soft tissue reconstruction? A ten-year experience of microsurgical reconstruction using 334 latissimus dorsi flaps from a universal donor site. Ann Plast Surg 2015;75:49-54.

9. Houston GC, Drew GS, Vazquez B, Given KS. The extended latissimus dorsi flap in repair of anterior abdominal wall defects. Plast Reconstr Surg 1988;81:917-24.

10. DeFranzo AJ, Pitzer K, Molnar JA, Marks MW, Chang MC, Miller PR, et al. Vacuum-assisted closure for defects of the abdominal wall. Plast Reconstr Surg 2008;121:832-9. 